\title{
Time Frequency Analysis of Higher Harmonic Generation in a Three Color Laser Pulse
}

\author{
Forouq Hosseinzadeh, Saeed Batebi* , and Mostafa Qadiri Soofi \\ Department of Physics, University of Guilan, Rasht, Iran \\ *Corresponding author: s_batebi@guilan.ac.ir
}

Received: Jan. 2, 2017, Revised: Feb. 23, 2017, Accepted: May. 16, 2017, Available Online: Oct. 28, 2017

\begin{abstract}
- high harmonic generation is a useful tool for the generation of short, intense attosecond pulses. In order to simulate high harmonic generation, we performed a numerical solution to the time dependent Schrödinger equation. by considering dipole approximation, we predicted generation of a 53 attosecond pulse. In order to see the time and frequency of emission of attosecond pulse, we exploit time frequency analysis. On the other hand, because of uncertainty between time and frequency, it would be of high importance whether which analysis is been applied. our studies show that Gabor analysis exhibits the least uncertainty between time and frequency components. And at least, we set the balance between time and frequency distribution by altering the window size.
\end{abstract}

KEYWORDS: Schrödinger equation, harmonic spectrum, isolated attosecond pulse, time frequency analysis.

\section{INTRODUCTION}

In last 50 years, much effort has been done to reduce laser pulse durations to achieve ultra fast phenomena [2]. From 1960 to 2010, pulse durations shortened about $10^{6}$ orders of magnitude [1]. So, attosecond science inherits the benefits of 50 years of research into concepts and approaches for fast measurement [2]. High harmonic generation( $H H G)$ and related processes are the gateway to the attosecond world. So HHG can be a useful tool to generate short coherent attosecond pulses. When atoms of rare gases are illuminated by intense $\left(10^{13}-10^{15} \mathrm{~W} / \mathrm{cm}^{2}\right)$ laser pulses the binding electron traps in a barrier which is made by the laser field and atomic potential. When the laser field approaches its maximum, electron tunnels through the barrier and freely starts oscillation in the laser field. By the next peak, laser field changes sign and electron bends its trajectory across the parent ion. As the electron gets closer to the atom, it recombines with the nucleus and emits a photon. This photon can be on the order of attosecond [3]. Attosecond pulses are the shortest bursts of light ever observed [2]. In intensities above $10^{18} \mathrm{~W} / \mathrm{cm}^{2}$ one approaches the relativistic regime. Harmonic spectrum has a special and global shape. It drops for the first few harmonics, making a flat region where all the harmonics have approximately the same intensity, and is labeled plateau region. The harmonic spectrum ends up with a sharp cut off. This is the point where emission of attosecond pulse takes place. As we control the harmonic spectrum well, the attosecond pulse becomes shorter. For higher frequencies, emission becomes more intense. So many tasks have been done to achieve higher cutoff frequencies and smoother and more efficient plateau. In [6]-[11] authors used chirped laser pulses in order to amplify the laser field. In other works researchers synthesized one or two laser pulses to the fundamental laser pulse, creating a multicolor laser pulse [8], [15], [24], [25], not only to amplify the field, but also to make the peaks of the fields further. As a result, the electron which is ionized in the first peak oscillates longer time in the laser field before recombination. So it takes much more energy and emits a more energetic 
photon while recombination. With high harmonic generation the problem is that, intense laser pulses are not available in all laboratories. The necessity of using intense laser pulses is to make the rare gases ionized. So, researchers followed two approaches to ionize rare gases instead. The first approach was to exploit plasmonic properties of metal nanostructure [14]-[22]. Researchers proved that bowtie nanoantennas are able to enhance the laser field up to 20-40 dB. So, instead of using a $10^{13} \mathrm{watt} / \mathrm{cm}^{2}$ intensity, in the vicinity of a bowtie nanoantenna, with a $10^{11} \mathrm{~W} / \mathrm{cm}^{2}$ laser pulse, high harmonic generation is feasible. The other approach is to pre ionize the atoms to prepare them in coherent excited states. In this way, electrons experience lower bounding potential, and as a result, they can be ionized by low intensity laser pulses.

From the perspective where we sit today, we are a long way far from any exact clarification about what attosecond is, and even how it is possible to achieve such a pulse in experiment. Because of this, it is so important to meticulously explore the structure of high order harmonics to know more and make this process more tangible. With having all of these in mind, in this work, we tried to perform the best spatiotemporal structure of high order harmonics.

\section{THEORETICAL STUdIES}

In normal conditions, atoms are in their initial states. So, for a Helium atom in normal condition, the electron will have a nonchronological wave function. Such a wave function is introduced by Hydrogen like atoms wave functions. But when such an atom is exposed to the illumination of a laser pulse its wave function varies in accordance to time. So, to see how the wave function changes, it is necessary to solve time dependent Schrödinger equation. The so called Schrödinger equation reads: $i \hbar \frac{\partial \psi(z, t)}{\partial t}=H \psi(z, t)$

where $\psi(z, t), H$, and $\hbar$ are respectively the atom wave function, the Hamiltonian of the target atom in the condition that it is illuminated by an intense laser field, and reduced Planck's constant. The Hamiltonian is as follows [4]

$H=\left(-\frac{1}{2 m} \frac{d^{2}}{d z^{2}}+V_{\text {atom }}(z)+V_{\text {int }}(z, t)\right)$

$U(z, t)=V_{\text {atom }}(z)+V_{\text {int }}(z, t)$

where $V_{\text {atom }}$ is the atomic potential with model potential [23] for Helium atom and $V_{\text {int }}$ is the potential that results from interaction of space with the laser field. Since our calculations are based on atomic units, thus, m equals to 1 . Also, interaction potential and atomic potential are in atomic units.

$V_{\text {atom }}=-\frac{Z_{c}+a_{1} e^{-a_{2} z}+a_{3} z e^{-a_{4} z}+a_{5} e^{-a_{6} z}}{Z}$

Table 1 Values of parameters of atomic potential of Eq. 4 [23].

Atom $Z_{c} \quad a_{1} \quad a_{2} \quad a_{3} \quad a_{4} \quad a_{5} \quad a_{6}$

\begin{tabular}{llllllll} 
Values & 1 & 1.23 & 0.66 & -1.32 & 1.23 & -0.23 & 0.48 \\
\hline
\end{tabular}

Here, $Z_{c}$ is the charge seen by the active electron asymptotically. The interaction potential will be:

$V_{\text {int }}(z, t)=E(t) z$

where $E$ is the electric part of the electromagnetic laser field which in this work is chosen as a synthesis of three electric fields with different wavelength and carrier envelope phases. For $i=1$, we'll have a monochrome laser pulse. In which $E_{01}$ is the amplitude of the laser pulse. The propagation of the laser pulse is prepared as a cosine function. $\omega_{1}$ and $\varphi_{1}$ are the frequency and phase of the laser field. The exponential function exhibits the 
envelope of the field. Due to the symmetry of the system, a monochromatic laser field will result in creation of odd high harmonics. On the other hand, as [25] clearly describes, a monochromatic laser pulse will result in a low frequency emission. To see higher frequencies, it is better to add second or third fields. In this way, the symmetry assigned to the system will be omitted. Wavelength and phase of these controlling fields can be adjusted in a way to make the amplitude of the laser pulse higher. Because of this, we chose a three color laser pulse.

$E(t)=\sum_{i=1}^{3} E_{0 i} \cos \left(\omega_{i} t+\varphi_{i}\right) \exp \left(-2 \ln 2\left(\frac{t}{\tau_{i}}\right)^{2}\right)$

Table 2 Values of electric field parameters of Eq. 6

\begin{tabular}{cccc}
\hline \hline parameters & $i=1$ & $i=2$ & $i=3$ \\
\hline$E_{0 i}\left(10^{14} \mathrm{~W} / \mathrm{cm}^{2}\right)$ & 0.75 & 1.5 & 0.75 \\
$\lambda_{i}(\mathrm{~nm})$ & 1600 & 1168 & 792 \\
$\varphi_{i}$ & 0 & $0.27 \pi$ & $0.64 \pi$ \\
$\tau_{i}(f s)$ & 16 & 16 & 16 \\
\hline
\end{tabular}

Laser field forces electron to accelerate. With Newtonian equation, we have $F=m a$, since all the computations in this work are based on atomic units, $m=1$, so that one can consider $F=a$, on the other hand $F=-\nabla U$ with $a$ being the induced dipole acceleration. According to Ehrenfest theory, the time dependent dipole acceleration is given by:

$a(t)=\left\langle\psi(z, t)\left|-\frac{d V_{a t o m}(z)}{d z}+E(t)\right| \psi(z, t)\right\rangle$

By computing the Fourier transformation of the dipole acceleration, the power spectrum of high harmonics is generated [23].

$$
p(\omega)=\int a(t) e^{-i \omega t} d t
$$

The attosecond pulse will be calculated by [23]:

$$
I(t)=\left|\sum_{q} a_{q} e^{i q \omega t}\right|^{2}
$$

where $a_{q}$ is given by $a_{q}=\int a(t) e^{-i q \omega t} d t \cdot \mathrm{q}$ is the harmonic order and is chosen from the harmonics near the cutoff region [4].

The Fourier transform has been the most commonly used tool for analyzing frequency properties of a signal, while after transformation, the information about time is lost and it's hard to tell where a certain frequency occurs. According to Eq. 8, Fourier transformation of time dependent dipole acceleration, gives the power spectrum of an atom. By filtering out some of the harmonics and calculating it's squared case, it gives the spectral intensity of the attosecond pulse which could be generated. Eq. 9 shows the time profile of the intensity of the generated attosecond pulse. It is clear that with the temporal profile of the attosecond pulse, we lose information about frequency and with the spectral profile of high harmonics nothing is understood about the temporal information of the attosecond pulse. But it is of high importance to see where a certain frequency occurs. So it would be beneficial to apply a time frequency analysis in order to observe the spatiotemporal structure of attosecond pulse. To achieve this goal, kinds of time frequency analysis techniques can be used. In the following, we explain more about time frequency analysis.

\section{III.RESULTS AND DISCUSSION}

After solving Schrödinger equation, it would be interesting to see the behavior of wave of wave function. Since wave function doesn't have physical meaning, we plot wave packet $\left(\psi^{2}\right)$, that explains the probability of presence of electron in a point. As a result of illumination of the laser field, the wave packet distributes in space and propagates in time. When it reaches the walls of the spatial window, it reflects and propagates toward the middle of the window and interacts with the propagating (not reflected) wave packet. So, these nonphysical and spurious reflections disturb the structure of the wave packet and lead to wrong results. To avoid such spurious 
and deleterious reflections from the spatial boundaries one can exploit two approaches: 1) choosing large spatial windows, 2) using absorbent potentials in the boundaries. The problem with the first approach is that, because of the limitation of our personal computer memories, this method takes so much time, albeit, if the computer was able to run the code. So the second approach seems better [31]:

$V_{\text {Absorb }}=\mid \begin{array}{ll}-i V_{0}\left(\frac{z-z_{1}}{z_{2}-z_{1}}\right)^{\alpha} & z_{1} \leq z \leq z_{2} \\ 0 & \text { otherwise }\end{array}$

where $z_{1}$ and $z_{2}$ define the range in which negative imaginary potential is operative. In our calculation $V_{0}$ and $\alpha$ are set to be 5 and 4 , respectively.

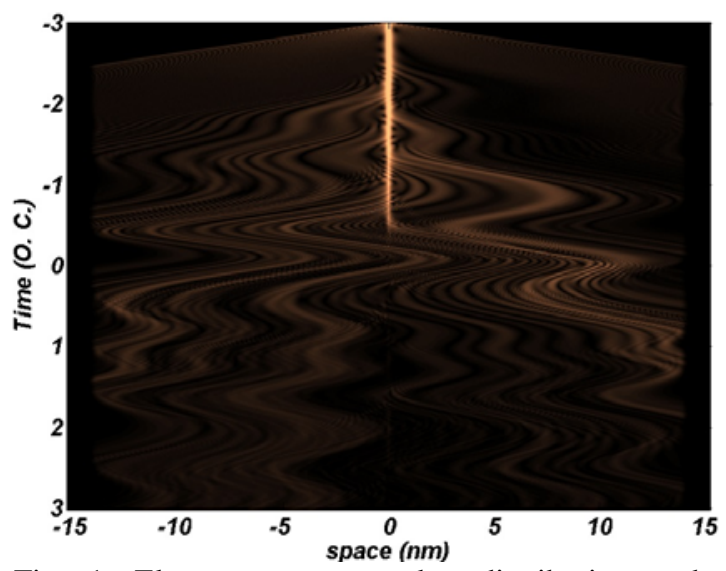

Fig. 1. Electrons wave packet distribution and propagation in space and time. O.C. stands for optical cycle.

The absorbing potential is added in potential term of the Hamiltonian. In Fig. 1, one can see the spatial distribution of wave packet distributes well in the time and space window. In walls of the space window, wave packet is absorbed by the negative imaginary potential. Thus, no reflection is observed on spatial boundaries . theoretically, by illuminating an intense laser field to the rare gases, high harmonics can be generated.

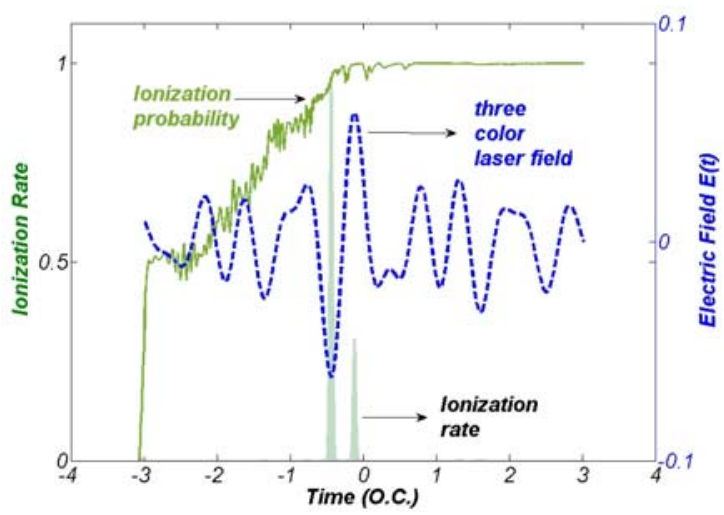

Fig. 2. electric field, ADK ionization rate and ionization probability. in this figure electric field corresponds to the right y axis . ionization rate and ionization probability correspond to the left y axis

In Fig. 2, one can see the three color laser field, ionization rate which is computed by ADK ionization rate principle [1], and the alteration of population of the ground state [4].

$$
p(t)=1-\left|\left\langle\psi(t) \mid \psi_{0}\right\rangle\right|^{2}
$$

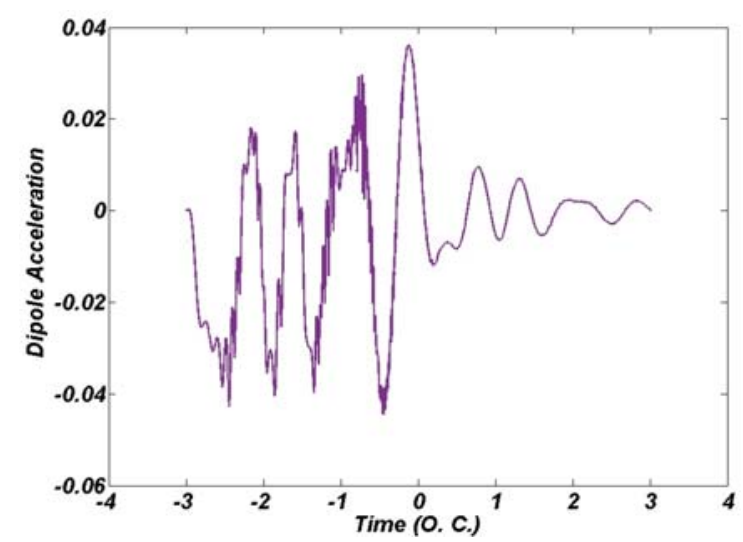

Fig. 3. Dipole acceleration.

The population alteration expresses that, before emission of the laser field, none of the atoms are in their new state $\psi(t)$ but when time goes on and laser field starts oscillation and population in this time dependent new state becomes greater. When the electric field reaches its maximum, ionization rate strongly takes place. So, in this region, population approximately concentrates on the new state. In other words, it can be deducted that in the point that the amplitude of the laser field is the 
greatest the probability of existence of electron in its new time dependent state is a hundred percent.

Now that the modification of wave packet from the first ground state is observed, it would be of interest to see the dipole acceleration behavior in time. Figure 3 shows dipole acceleration.

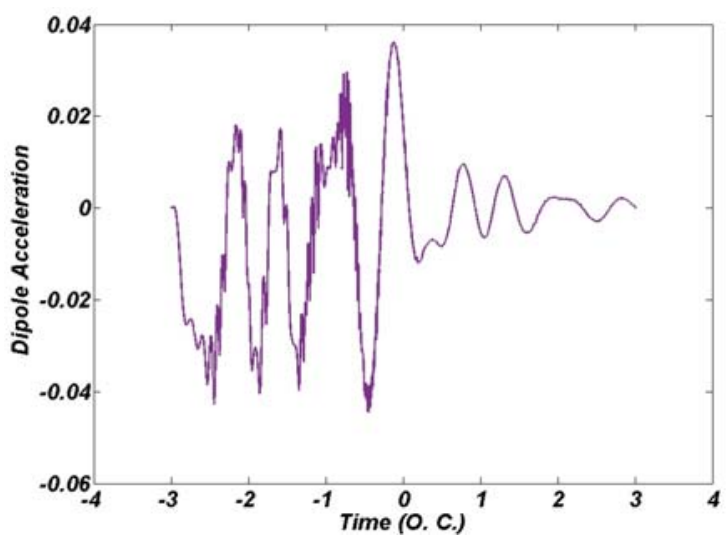

Fig. 4. Dipole acceleration

Equation 8 gives the spectral profile of the dipole acceleration. By filtering out some of the harmonics, $a_{q}$ transforms the time dependent dipole acceleration in to spectral range. Again by computing the Fourier transformation of $a_{q}$ on the same harmonics, and computing its squared form, the temporal profile of dipole acceleration on specific harmonics, is achieved. By equation 8 one can see on which harmonic emission takes place. But in this equation no information is achieved about the time profile of the emission. We only know the emissions frequency. But it is so important to know both the time and frequency of an emission. In order to observe spatiotemporal structure of the dipole acceleration, it would be interesting to apply a time frequency analysis to the dipole acceleration. Generally, to observe the spatiotemporal distribution of a signal, its Fourier transformation must be multiplied by a temporal window function (WF).

$$
a(\Omega, t)=\int d t^{\prime} a\left(t^{\prime}\right) W F\left(t^{\prime}-t\right) \exp \left(i \Omega t^{\prime}\right)
$$

The WF mimics the action of an experimental spectral filter that selects only a range of XUV frequencies [2].

Time frequency analysis computes the integrant of the Fourier transformation of a portion of the signal $a\left(t^{\prime}\right)$ which is limited by the.WF In other words, WF chooses a part of the signal $a\left(t^{\prime}\right)$ to be transformed.

It is notable that, there is always uncertainty between the time and the frequency according to Heisenberg uncertainty. Meaning that time and frequency cannot contemporarily been measured with high precision. whatever one gets closer to measure time components of the analysis, it loses information about frequency and vice versa. Thus, it is essential to exploit a method to reduce this uncertainty. It is of high importance to choose the width of this window in a way that it lessens the uncertainty between time and frequency. But before trying to find the best size for the $W F$, it is necessary to choose the WF 's shape. Several works have been done to choose the best temporal WF in order to gain the least uncertainty in both time and frequency components. In this work, we tried the rectangular $W F$, the Hamming $W F$, and Gaussian WF.

A rectangular $W F$, as it is clear from its name, has a table top shape meaning that it possesses only two values, zero or one. In the first step, of our study, we've fixed the $W F$ 's width equal to $2 \pi$. it approximately equals 6 atomic units. In our case, as is introduced in table 2, the laser pulse has a 664 atomic units time duration. A 6 atomic units wide $W F$ seems so narrow in comparison with the 664 a. u. time duration. So, a rectangular WF equals 1 only in 6 a. u. and in other times, it is zero. Now, by substituting these 1 or 0 values in Eq. 12, we can have the transformation only in that 6 atomic unit that the WF equals 1 . in other times, it would be zero. In the points that WF equals 1, Eq. 12 only computes the Fourier transformation of $a\left(t^{\prime}\right)$. So, the separation is done only for frequency components rather than temporal components. Figure 4 shows a 
time frequency analysis for the dipole acceleration by using a rectangular WF. By focusing on this figure, we can see that apparently, the temporal information of the signal is approximately lost and the function only separated the frequency components.

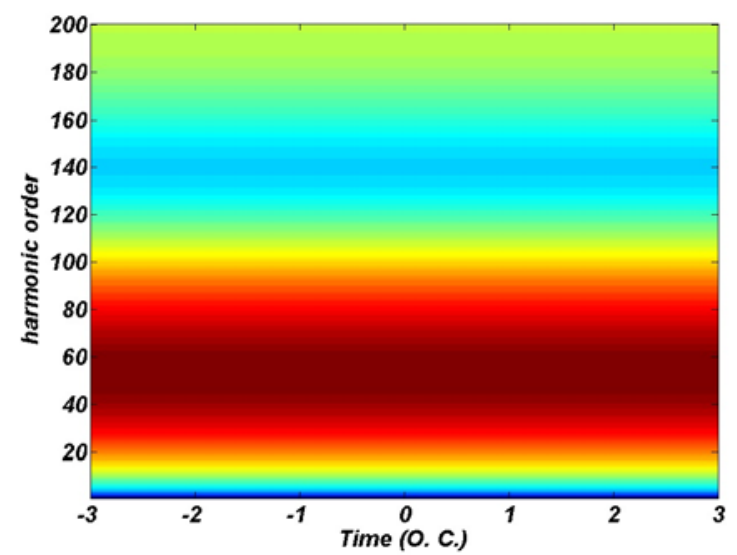

Fig. 5. Time frequency analysis of dipole acceleration by considering the WF as a rectangular WF.

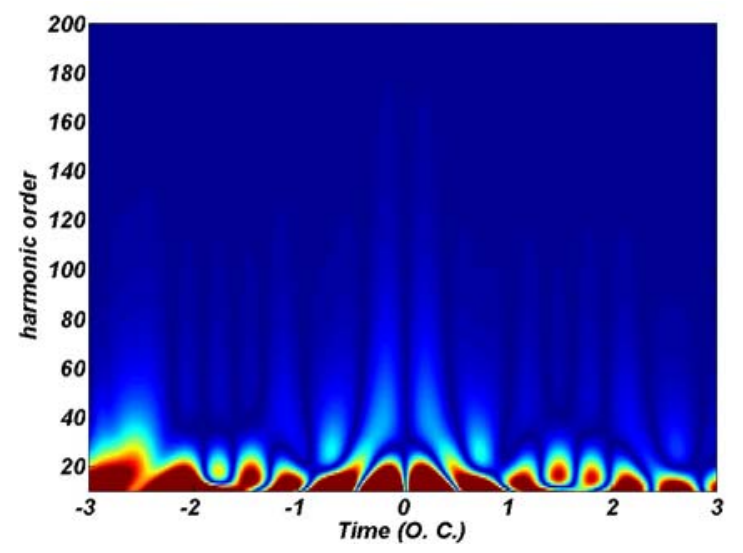

Fig. 6. Time frequency analysis of dipole acceleration by considering the WF as a Hamming WF.

So, we can see that, rectangular WF shows a high uncertainty between time and frequency. In the next step, we tried Hamming WF. The result is shown in Fig. 5. in this figure, one can clearly see that the uncertainty between time and frequency is less than rectangular one. Since the distribution of the signal in time and frequency is completely discrete. But in this case, a specific result cannot be achieved. So, in the next step, we calculate the time frequency analysis driven by a Gaussian WF. Just like a rectangular $W F$, the function equals zero in most of the time and in other times it gradually increases. So, since this function's value is not constant in respect to time, its result is different from the rectangular one.

With a Gaussian WF, the time frequency analysis approaches to Gabor time frequency analysis [5] which is in the form:

$a_{G}(\Omega, t)=\int d t^{\prime} a\left(t^{\prime}\right) \frac{\exp \left[\frac{-\left(t^{\prime}-t\right)^{2}}{2 \sigma^{2}}\right]}{\sigma \sqrt{2 \pi}} \exp \left(i \Omega t^{\prime}\right)$

The WF acts like a switch which turns $\mathrm{ON}$ and $\mathrm{OFF}$. We call the window function OFF whenever it equals zero, unless it is called ON. Calculations are done for parameters $\Omega, t$ and $t^{\prime} . \Omega$ in Vol.ves the whole harmonics of the related power spectrum (containing the first to the last harmonic orders), $t$ and $t^{\prime}$ are both within laser pulse duration. $t$ is the time that changes the points where $W F$ is ON. In order to explain what is the parameter $t$ responsible for, let us ignore it, and see what happens if this parameter doesn't exist. In this case, calculation is done for only a portion of the signal $a\left(t^{\prime}\right)$ that is limited with the Gaussian WF. In this case, in all of the variations of $t^{\prime}$ and $\Omega$, the integrant in equation 13 is done for a specific portion of the signal $a\left(t^{\prime}\right)$. Since the width of such a WF is so small in comparison with the whole induced dipole acceleration time, this small element of the signal $a\left(t^{\prime}\right)$ gives no sufficient and verified information about the spatiotemporal profile of induced dipole acceleration. So in order to use a larger element of the signal $a\left(t^{\prime}\right)$, it seems it would be good to make the WF wider. But, choosing a wider $W F$ will not solve this problem because of the uncertainty between time and frequency. In order to take the whole signal into calculations, it is necessary to change the 
place where $W F$ is ON with the help of changing the values of a parameter like $t$. For each $\Omega^{\text {th }}$ frequency and $t^{\text {th }}$ time, the integration is taken over variation of the time $t^{\prime}$. When time $t$ goes on, WF becomes $\mathrm{ON}$ in later time. So, the points of the dipole acceleration which take part in Gabor transformation in previous time differ from the next time. Thus, $a_{G}(\Omega, t)$ can be computed for every points of signal $a\left(t^{\prime}\right)$.

Figure 6-b shows the spatiotemporal profile of signal $a\left(t^{\prime}\right)$. See how great the time and frequency components are separated.

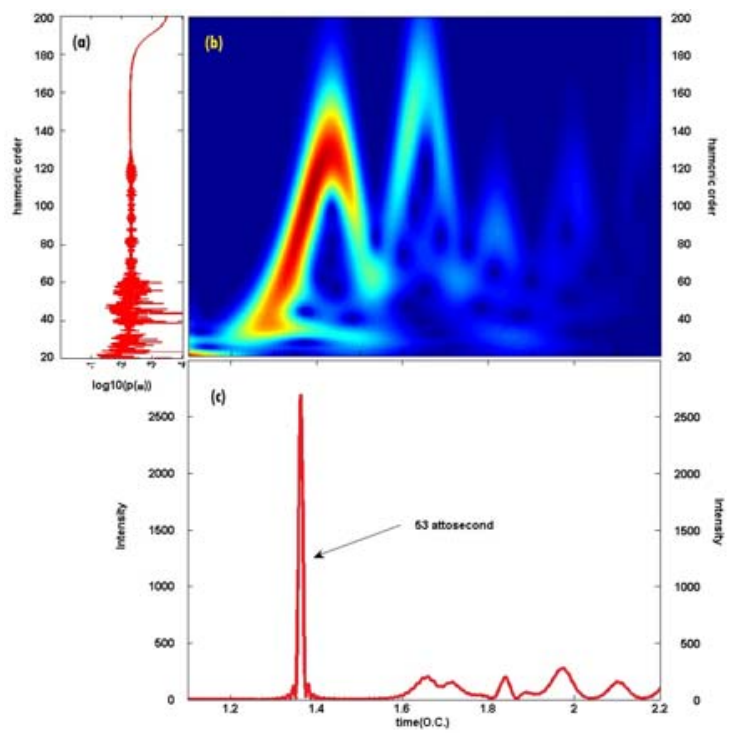

Fig. 7. (a) Spectral profile of high harmonic generation (HHG). (b) Time frequency analysis of dipole acceleration by considering the WF as a Gaussian WF. (c) Time profile of hhg that exhibits the synthesis of an isolated 53 attosecond pulse.

By agreement with C. Chandre et al. [32], our simulation proves that with a Gaussian WF uncertainty between time and frequency is the least [32]. The Gaussian function has all sorts of nice properties, among which

1) The Fourier transformation of the Gaussian function is again a Gaussian

2) The Gaussian function minimizes the product of time duration and spectral bandwidth.

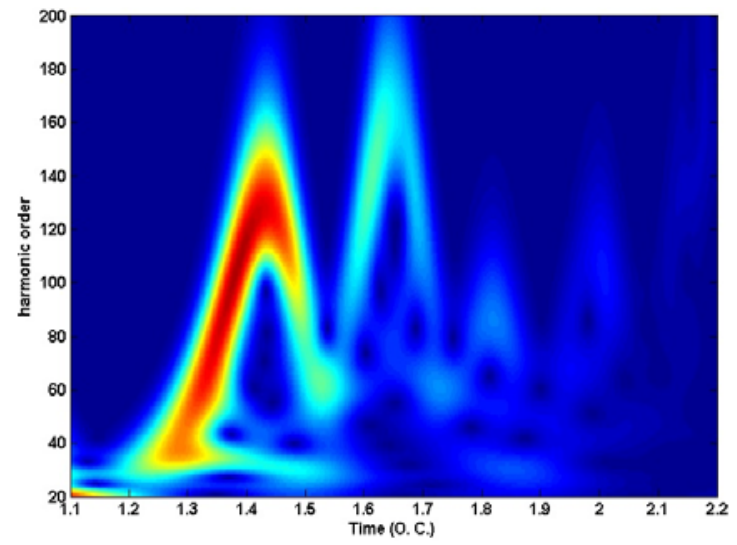

Fig. 8. Gabor transformation of the induced dipole acceleration by setting $\sigma=2 \pi$.

Figure 6 shows spectral profile and time profile of HHG in zones (a) and (c) respectively. Zone (b) shows time frequency analysis of HHG. The power spectrum of high order harmonics is computed by Eq. 8 and is shown in Fig. 6-a. in this figure, one can clearly see that the power spectrum starts to get smoother from $80^{\text {th }}$ order up to $180^{\text {th }}$ order that is the cutoff of the power spectrum and corresponds to the peak of the time frequency distribution (in part (b)). The Gabor transformation of dipole acceleration that is given by Eq. 13 is depicted in zone b. it asserts that each frequency can occur on two different times. It means that electrons that take part in HHG process may pass two separated energy paths. In this work short path is more dominant. In the region that distribution of HHG is more dominant, emission takes place. By exploring time profile of HHG that is computed by Eq. 9 by filtering harmonics near cutoff region, and isolated 53 as pulse is generated. This pulse measures the width of the emitted photon. It is of principle to point that according to Fig. 2 ionization rate is maximum into time steps. The electrons that are ionized in Fig. 2 recombine in later times in Fig. 6-b. but the only emission that leads to generation of attosecond pulse is the one that emits between times 1.37-1.4. fluctuations of the intensity in Fig. 6-c shows the intensity of their corresponding emission in zone b. before, it was mentioned that $\sigma$ which is the width of $W F$ plays an important role on the resolution of spectral and temporal components. 


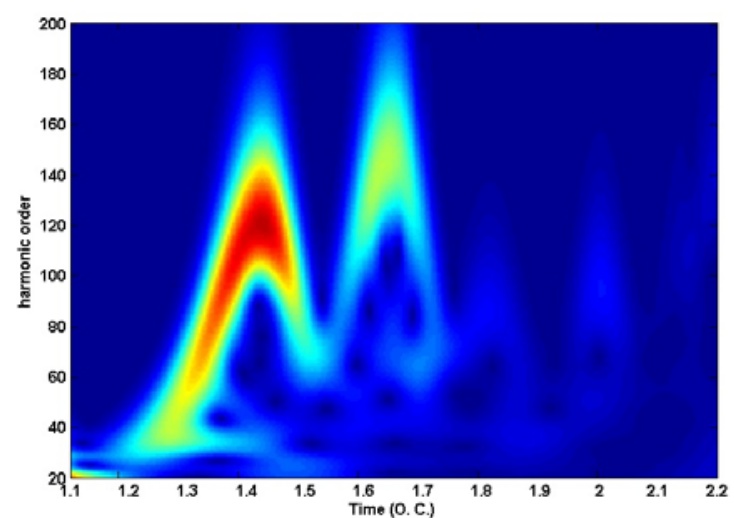

Fig. 9. Gabor transformation of the induced dipole acceleration by setting $\sigma=1 / 3 \omega_{1}$ with $\omega_{1}$ being the central laser frequency.

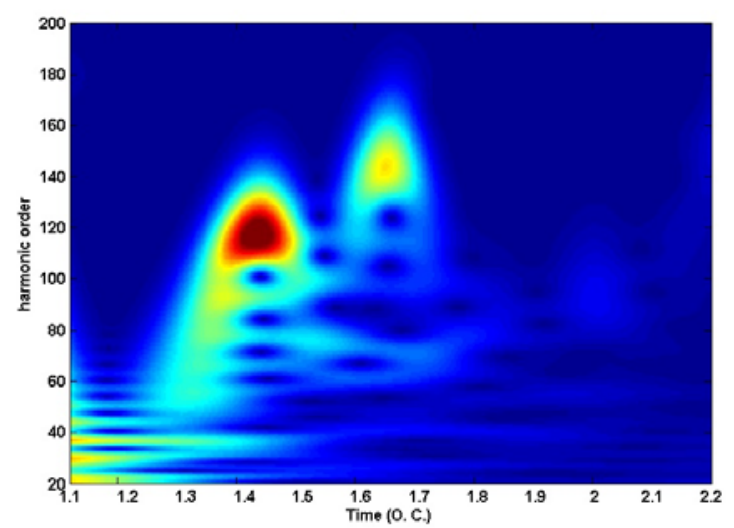

Fig. 10. Gabor transformation of the induced dipole acceleration by setting $\sigma=15$.

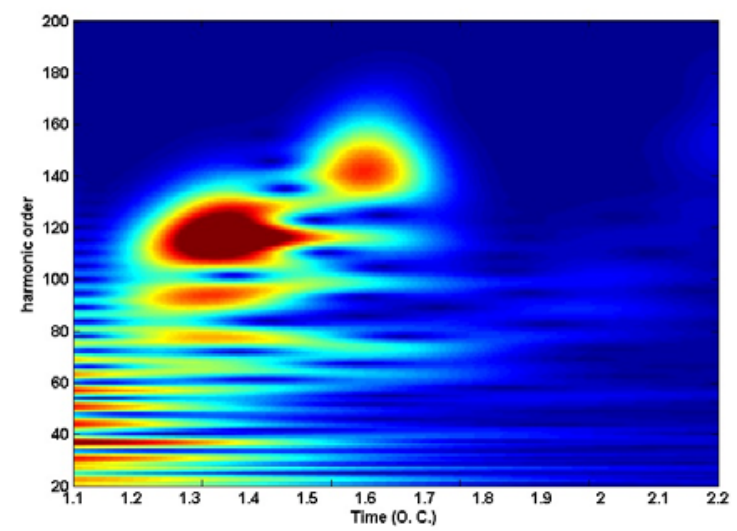

Fig. 11. Gabor transformation of the induced dipole acceleration by setting $\sigma=30$.

In previous works, authors choose different $\sigma$ values. In this work, we tried some of them. Like as, $\sigma=1 / 3 \omega_{1}$ [5] with $\omega_{1}$ being the central laser frequency of $\sigma=2 \pi$ [27], $\sigma=15$ [12], and $\sigma=30$ [19]. By comparing the results of these $\sigma$ values, it is deducted the larger the $\sigma$ parameter is, the worst the resolution will be. By the large $\sigma$ s the WF becomes wider, so, the distribution of signal expands in time and shrinks in the spectral range. Thus, the information about time will be lost. So, $\sigma=2 \pi$ is chosen to be the most optimum window size in this work.

\section{IV.CONCLUSION}

In this work, we've solved 1D time dependent Schrödinger equation with the purpose of generating isolated attosecond pulses. We numerically simulated high order harmonic generation and then by filtering 60 harmonics near the cutoff region $\left(120^{\text {th }}-180^{\text {th }}\right)$ we extracted an isolated 53 attosecond pulse. We succeed to separately observe time profile of induced dipole acceleration we could see the frequency of the emitted photon which is the cutoff frequency. But the problem with the spectral profile of the induced dipole acceleration was that it didn't give us any information about the time that such a photon is emitted. Similarly in time profile of the induced dipole acceleration, we might know nothing about frequency. Thus it is important to see when a certain frequency occurs. In order to meticulously explore the structure of HHG, we performed a time frequency analysis by using different temporal window functions. At last, we deducted that a Gaussian window function exhibits the least uncertainty between time and frequency components. To balance the resolution of the spatiotemporal distribution of HHG we tried different WF sizes and at last, we found $\sigma=2 \pi$ as the best size for this parameter.

\section{REFERENCES}

[1] Z. chang, Fundamentals of Attosecond Optics, Taylor and Francis, 2011.

[2] L. Plaja, R. Torres, and A. Zaïr, Attosecond Physics, Springer Series in Optical Sciences, 2013. 
[3] P.B. Corkum and F. Krausz, "Attosecond science," Nature Phys. Vol. 3, pp. 381-387, 2007.

[4] V.T. Platonenko, A.F. Sterjantov, and V.V. Strelkov, "Decrease of high harmonic generation yield in the barrier-suppression regime," Laser Phys. Vol. 13, pp. 443-449, 2003.

[5] C.C. Chirila, I. Dreissigacker, E.V. van der Zwan, and M. Lein, "Emission times in high order harmonic generation," Phys. Rev. A, Vol. 81, pp. 033412 (1-7), 2010.

[6] L. L.Yong, D. H.Chuan, and H. B.Tao, "High orde harmonic generation with a two color laser pulse," Chin. Phys. B, Vol. 21, pp. 033202 (1-5), 2012.

[7] H. Du and B. Hu, "Broad band supercontinuum generation method combining mid infrared chirped pulse modulation and generalized polarization gating," Opt. Express, Vol. 18, pp. 25958-25966, 2010.

[8] T. Nemati Aram, S. Batebi, and M. Mohebbi, "Numerical simulation of an intense isolated Attosecond pulse by a chirped two-color laser field," Int. J. Opt. Photon. Vol. 6, No. 1, pp. 312, 2012.

[9] J.J. Carrera and Sh. Chu, "Estension of high order harmonic generation cutoff via coherent control of intense few cycle chirped laser pulses," Phys. Rev. A, Vol. 75, pp. 033807 (15), 2007.

[10] P. Zou, Zh. Zeng, Y. Zheng, Y. Lu, P. Liu, R. $\mathrm{Li}$, and $\mathrm{Zh}$. $\mathrm{Xu}$, "Coherent control of broad band isolated attosecond pulses in a chirped two color laser field," Phys. Rev. A, Vol. 81, pp. 033428, 2010.

[11] H. Du, L. Luo, X. Wang, and B. Hu, "Isolated attosecond pulse generation from pre-excited medium with a chirped and chirped-free two color field," Opt. Express, Vol. 20, pp. 97139725, 2012.

[12] M. Mohebbi, "Controlling the ionization and recombination rates of an electron in pre excited ions to generate an intense, isolated sub-4-as pulse in a multi cycle regime," Phys. Rev. A, Vol. 91, pp. 023835, 2015.

[13]M. Mohebbi, "A strong single attosecond pulse generation from an atomic superposition state with chirped laser fields," Optik, Vol. 125, Issue 15, pp. 3818-3821, 2014.
[14] S. Kim, J. Jin, Y. J. Kim, I. Y. Park, Y. Kim, and S. W. Kim, "High harmonic generation by resonant plasmon field enhancement," Nature, Vol. 453, pp. 757-760, 2008.

[15] X. Shan, D.H. Chuan, X. Yue, and H.B. Tao, "Generation of isolated attosecond pulses in bowtie shaped nanostructure with three color spatially inhomogeneous field," Chin. Phys. B, Vol. 24, pp. 054210 (1-8), 2015.

[16] M.F. Ciappina, S.S. Acimovic, T. Shaaran, J. Biegert, R. Quidant, and M. Lewenstein, "Enhancement of high order harmonic generation by confining electron motion in plasmonic nanostructures," Vol. 20, Issue 24, pp. 26261-26274, 2012.

[17]A. Husakou, S.J. Im, and J. Herrmann, "Theory of plasmon enhanced high harmonic generation in the vicinity of metal nanostructures in nobel gases," Phys. Rev. A, Vol. 83, 043839 (1-5), 2011.

[18] H. Zhong, J. Guo, W. Feng, P. Ch. Li, and X. Sh. Liu, "Comparison of high harmonic generation and attosecond pulse from 3D Hydrogen atoms in three kinds of inhomogeneous fields," Vol. 380, Issue 1-2, pp. 188-193, 2016.

[19] X. Cao, Sh. Jiang, Ch. Yu, Y. Wang, L. Bai, and $\mathrm{R}$. Lu, "Generation of isolated sub-10-as pulses in spatially inhomogeneous two color field", Vol. 22, Issue 21, pp. 26153-26161, 2014.

[20] Y.-Y. Yang, A. Scrinzi, A. Husakou, Q.-G. Li, S.L. Stebbings, F. Süßmann, H.-J. Yu, S. Kim, E. Rühl, J. Herrmann, X.-C. Lin, and M.F. Kling, "High harmonic and single attosecond pulse generation using plasmonic field enhancement in ordered arrays of gold nanoparticles with chirped laser pulses," Opt. Express, Vol. 21, pp. 2195-2205, 2013.

[21] T. Shaaran, M.F. Ciappina, R. Guichard, J.A. Perez- Hernandez, M. Arnold, T. Siegel, A. Zair, and M. Lewenstein, "High order harmonic generation by enhanced plasmonic near fields in metal nanoparticles," Phys. Rev. A, Vol. 87, pp. 041402(1-6), 2013.

[22] Y. Chou, P. Ch. Li, T.S. Ho, and S.I. Chu, "Generation of an isolated few attosecond pulse in optimized inhomogeneous two color field," Phys. Rev. A, Vol. 92, pp. 023423 (16), 2015. 
[23] X M Tong and C D Lin, "Empirical formula foe static field ionization rates of atoms and molecules by lasers in the barrier suppression regime," J. Phys. B: At. Mol. Opt. Phys. Vol. 38, pp. 2593-2600, 2005.

[24] L. Fei, W. Guo-Li, Z. Song-Feng, and Z. XiaoXin, "Synthesis of multi-color, long laser pulses for strong attosecond pulse generation," Chin. Phys. Lett. Vol. 32, pp. 014210 (1-4), 2015.

[25] P.Ch. Li, I.L. Liu, and Sh.I Chu, "optimization of three color laser field for the generation of single altra short attosecond pulse", Opt. Express, Vol. 19, pp. , 23857-23866, 2011.

[26] J.J. Carrera, X.M. Tong, and Sh.I. Chu, "Creation and control of a single coherent attosecond XUV pulse by few cycle intense laser pulse," Phys. Rev. A, Vol. 74, pp. 023404 (1-7), 2006.

[27] A. Ahmadi, A. Maghari, H. Sabziyan, A.R. Niknam, and M. Vafaee, "Effects of nuclear motion on high order harmonic generation of $\mathrm{H}_{2}^{+}$in intense ultra-short laser pulses," Phys. Rev. A, Vol. 90, pp. 043411 (1-7), 2014.

[28] Ph.E. Batson, "Plasmonic modes reveald," Science, Vol. 335, pp. 47-48, 2012.

[29] J.M. Reed, Light matter interactions of plasmonic nanostructures, PHD Thesis, B.S. University of Central Florida, 2013.

[30]C. Sonnichsen, Plasmons in metal nanostructures, $\mathrm{PhD}$ thesis, LudwigMaximilians-University, Munich, 2001.

[31] H. Sabziyan and H. Ebadi, "Ionization of a 1D model of $\mathrm{H}_{2}^{+}$from different states in intense laser field," Iranian J. Science Technol. Trans. A, Vol. 33, No. A1, pp. 87-102, 2009.

[32] C. chandre, S. Wiggins, and T. Uzer, "Time frequency analysis of chaotic systems," Vol. 181, pp. 171-196, 2003.

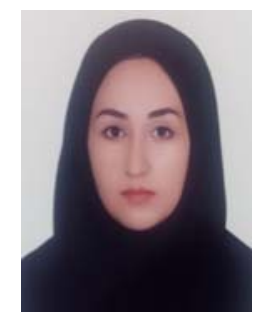

Forouq Hosseinzadeh was born in Rasht, Guilan, Iran. She got her bachelor degree in optics and laser engineering from Bonab University in Bonab, Bonab, in 2014 and continued her studies in master of Photonics in Guilan University and graduated in 2017. Her research interests are mostly based on the prediction of generating ultra-short attosecond pulses.

She is the author of one international journal and 5 conference articles. She is also the coauthor of 7 conference paper.

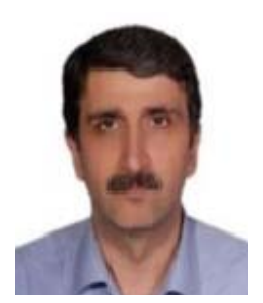

Saeed Batebi has received his BSc, MSc, and $\mathrm{PhD}$ degree respectively in applied physics from Ferdowsi University, Mashhad, Iran, Physics from Sharif University of Technology and laser physics from Moscow State University, Moscow, Russia Federation in 1987, 1990, and 2003.

$\mathrm{He}$ is an academic member of Guilan University, Guilan, Iran from 1992. his research interests are nonlinear optics, laser plasma interaction, higher harmonic generation and attosecond science.

Dr. Batebi is the author and coauthor of many journal and conference papers.

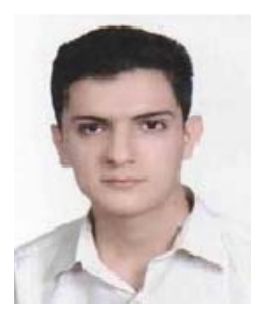

Mostafa Qadiri Soofi has received his BSc and MSc degrees in optics and laser engineering from Malek Ashtar University, Tehran, Iran, and Photonics from Guilan 
University, Guilan, Iran, in 2013 and 2016. Now he is a $\mathrm{PhD}$ candidate in photonics at Shahid Beheshti University, Tehran, Iran.
He is the author of one journal and 15 conference papers. 
THIS PAGE IS INTENTIONALLY LEFT BLANK. 